\title{
Desempenho Termoenergético de Edificações Sob a Ótica das Mudanças Climáticas: Uma Revisão Sistemática
}

\section{Buildings Thermoenergetic Performance from the Viewpoint of Climate Change: A Systematic Review}

\author{
${ }^{1}$ Emeli Lalesca Aparecida da Guarda, ${ }^{2}$ Luciane Cleonice Durante, ${ }^{3}$ Ivan Júlio Apolônio Callejas \\ ${ }^{1}$ Doutoranda do Programa de Pós-graduação em Arquitetura e Urbanismo, Faculdade Federal de Santa Catarina, \\ UFSC, Campus UFSC - Trindade, Florianópolis-SC (emeliguarda@ gmail.com) \\ ${ }^{2}$ Doutora em Física Ambiental, Professor Adjunto IV da Faculdade de Arquitetura, Engenharia e Tecnologia, \\ Universidade Federal de Mato Grosso, Av. Fernando Correa da Costa, 2367, FAET, Cuiabá-MT, \\ (luciane.durante@hotmail.com) \\ ${ }^{3}$ Doutor em Física Ambiental, Professor Adjunto IV da Faculdade de Arquitetura, Engenharia e Tecnologia, \\ Universidade Federal de Mato Grosso, Av. Fernando Correa da Costa, 2367, FAET, Cuiabá-MT, E-mail:
}

(ivancallejas1973@gmail.com)

\begin{abstract}
RESUMO: Este artigo parte da premissa de que as alterações climáticas registradas são consequências do aquecimento global, sendo seus efeitos passíveis de mensuração no ambiente construído. As condições internas de conforto e habitabilidade nas edificações têm sido estudadas com amparo de simulação computacional de cenários futuros, tomando-se por base arquivos climáticos que incorporam os efeitos do aquecimento. O objetivo deste trabalho é elaborar um panorama das publicações científicas acerca das projeções de aumento da temperatura interna e do consumo de energia elétrica em edificações, considerando os parâmetros do Quarto Relatório (AR4) do Painel Intergovernamental sobre Mudanças Climáticas (IPCC). Utilizou-se da metodologia de revisão sistemática para reconhecer os critérios considerados no estabelecimento dos cenários futuros (2020, 2050 e 2080) e a distribuição espacial dos estudos sobre o tema. Os resultados apontam para uma grande variabilidade da demanda de energia, justificada pelas diferenças nos critérios adotados, nas tipologias construtivas e na localização dos edifícios pesquisados. Um recorte para o setor residencial brasileiro, revela que apesar das disparidades entre as demandas previstas nos cenários de aquecimento global, todas as provisões indicam elevação de consumo energético para manutenção da habitabilidade nas zonas bioclimáticas brasileiras, sendo, portanto, indispensável considerar a resiliência das edificações como forma de estratégia de adaptação.
\end{abstract}

Palavras Chave: Aquecimento Global. Resiliência de edificações. EnergyPlus.

ABSTRACT: This article is based on the premise that the registered climate changes are consequences of global warming, and their effects can be measured in the built environment. The internal conditions of comfort and habitability in buildings have been studied with the help of computer simulation of future scenarios, based on climatic files that incorporate the effects of heating. The objective of this work is to elaborate an overview of the scientific publications about the projections of an increase of the internal temperature and the consumption of electric energy in buildings, considering the parameters of the Fourth Report (AR4) of the Intergovernmental Panel on Climate Change (IPCC). The systematic review methodology was used to recognize the criteria considered in the establishment of future scenarios (2020, 2050 and 2080) and the spatial distribution of studies on the theme. The results point to great variability in the demand for energy, justified by the differences in the adopted criteria, in the construction typologies and in the location of the surveyed buildings. A cut for the Brazilian residential sector, reveals that despite the disparities between the demands foreseen in the global warming scenarios, all provisions indicate an increase in energy consumption to maintain habitability in Brazilian bioclimatic zones, therefore it is essential to consider the buildings resilience as an adaptation strategy.

Keywords: Global warming. Building resilience. EnergyPlus.

\section{INTRODUÇÃ̃O}

Um dos principais desafios a serem enfrentados pela sociedade do século XXI é o de promover ações mitigadoras das mudanças climáticas. Estudos apontam que entre o final do século XIX e início do século XX, as temperaturas apresentavam comportamento praticamente invariável. Entretanto, a partir da década de 1920, há uma tendência crescente 
das temperaturas, com registro de aumento de $0,8^{\circ} \mathrm{C}$ no ano de 2000 , em relação às médias do período de 1961 a 1990 (CASAGRANDE, 2013).

Diante dessa realidade, faz-se necessário conhecer o comportamento das condições de tempo atmosférico, impulsionando estudos climatológicos, para fins de diagnósticos e prognósticos, e incentivar o avanço de modelos que atendam as previsões de tempo e clima (REPELLI e ALVES, 1996; XAVIER et al., 2000; LUCIO et al., 2010).

O Painel Intergovernamental sobre Mudanças Climáticas (IPCC) tem a função de compor o conhecimento científico referente às mudanças climáticas motivadas pelas ações antrópicas, seus impactos e riscos para o meio ambiente e humanidade. O IPPC foi criado em 1988, pela Organização Meteorológica Mundial (OMM) e pelo Programa das Nações Unidas para o Ambiente Construído (PNUMA). Desde então, tem publicado relatórios científicos sobre as alterações climáticas no mundo: o primeiro, lançado em 1990 (AR1); o segundo, em 1995, foi um importante instrumento para a adoção do Protocolo de Kyoto, em 1997 (AR2); o terceiro, em 2001 (AR3); o quarto, em 2007 (AR4) e o quinto e mais recente, em 2013 (AR5). A partir desses relatórios, os cientistas das mais variadas áreas de conhecimento fundamentam as pesquisas sobre o comportamento dos ecossistemas terrestres.

O Quarto Relatório de Avaliação (AR4) - Special Report Emission Scenarios (SRES) traz os cenários de emissões de gases de efeito estufa. Os modelos são divididos em quatro famílias, denominados como cenários $\mathrm{A} 1, \mathrm{~A} 2, \mathrm{~B} 1$ e $\mathrm{B} 2$, onde ' $\mathrm{A}$ ' e ' $\mathrm{B}$ ' significam baixo e alto comprometimento com o desenvolvimento sustentável, e ' 1 ' e '2', a integração ou fragmentação regional, respectivamente (SRES, 2010).

Os cenários de emissão de gases de efeito estufa desenvolvidos pelo IPCC são condições futuras com históricos demográficos, sociais, econômicos e tecnológicos similares. Os cenários de classificação 'A' são considerados 'pessimistas', sendo que A1 representa um futuro de rápido crescimento econômico e não sustentável, dividido em A1F1, com uso intensivo de combustíveis fósseis; A1T, com fontes não fósseis; A1B, com equilíbrio entre as fontes e A2, que representa um mundo fragmentando, não sustentável, com alto índices de população. Os cenários de classificação 'B' são considerados 'otimistas', sendo que B1 representa um mundo integrado e sustentável, com implantação de tecnologias limpas e a busca de soluções globais para problemas econômicos, excluindo as alterações climáticas e B2, trata de cenário semelhante ao B1, porém com iniciativas das nações para balancear objetivos econômicos, sociais e ambientais. Considerando-se o ambiente construído, os modelos previsionais de temperatura interna das pesquisas sobre os impactos das mudanças climáticas se utilizam do relatório AR4.

O desempenho termoenergético dos edifícios depende do clima ao qual estão expostos (WILDE e COLEY, 2012) e o aumento da temperatura média global impõe impactos aos edifícios e, consequentemente, aos seus usuários (CASAGRANDE, 2013). Assim, pesquisas que abordam esse tema na escala do ambiente construído têm atuado no prognóstico das temperaturas urbanas por meio de modelos matemáticos (DU; EDGE; UNDERWOOD, 2011; KERSHAW; EAMES; COLEY, 2011; ROBERT; KUMMERT, 2012) e, mais recentemente, têm focado nas simulações de desempenho termoenergético de edifícios mediante os efeitos do aquecimento, obtendo como resultados condições de conforto e segurança dos ambientes internos consumo de energia elétrica (WANG et al., 2017; SONG e YE, 2017) e estratégias de projeto passivas para proporcionar condições adequadas de habitabilidade, apontando os estudos de resiliência como uma das formas de mitigação (CASAGRANDE e ALVAREZ, 2013; TRIANA et al., 2016; INVIDIATA e GHISI, 2016; FAHMY et al., 2017; SABUNAS e KANAPICKAS, 2017, JORGE et al., 2018).

A ferramenta de simulação computacional mais difundida nas simulações de cenários futuros de desempenho do ambiente construído é a Climate Change World Weather File Generator (CCWorldWeatherGen), que faz uso do método Morphing. 
Segundo Belcher, Hacker e Powell (2005), a metodologia Morphing parte de um conjunto de dados climáticos históricos referentes ao período de 1961-1990, adotado pelo fato de não conter influência do aquecimento global. Fahmy et al. (2014) acrescenta que o método utiliza as anomalias climáticas para mensurar novos dados meteorológicos que incorporem as projeções dos Modelos de Circulação Geral (MCG) desenvolvidos no Reino Unido.

Desta forma, têm-se arquivos climáticos com e sem a influência do aquecimento global, ambos no formato Test Reference Year (TRY) representa um ano (8.760 horas) de dados médios para uma região ou local específico, sem conter extremos de temperatura e abrange as variáveis de Temperatura de Bulbo Seco $\left({ }^{\circ} \mathrm{C}\right)$, Temperatura de Orvalho $\left({ }^{\circ} \mathrm{C}\right)$, Umidade Relativa do Ar (\%), Direção $\left(^{\circ}\right)$ e Velocidade do Vento (m/s), Nebulosidade (décimos), Pressão Barométrica (KPa) e Radiação Solar (Wh/m²). No Brasil, Goulart (1993) disponibiliza arquivos climáticos TRY para 14 cidades brasileiras.

A ferramenta CCWorldWeatherGen foi desenvolvida por "Sustainable Energy Research Group" (SERG), da Universidade de Southampton, no Reino Unido, juntamente com a Microsoft ® (JENTSCH, BAHAJ e JAMES, 2013). Permite a geração dos arquivos climáticos representativos de mudanças climáticas futuras no formato TRY anteriormente descrito, originando arquivos com a extensão Energy Plus Weather (EPW) para simulação de desempenho de edificações.

É disponibilizada em formato de planilha eletrônica permitindo a integração de arquivos de extensão EPW ao Modelo Climático Global (MCG) "Hadley Centre Coupled Model version 3" (HadCM3), que é um modelo acoplado oceano-atmosfera, com resolução espacial de $2,5^{\circ} \times 3,75^{\circ}$ (latitude e longitude, na região do Equador) e sua representação produz uma com resolução de 96 x 76 grids ou $417 \mathrm{~km}$ x $278 \mathrm{~km}$, que é reduzido para $296 \mathrm{x}$ $278 \mathrm{~km}$ em latitudes $45^{\circ}$ Norte ou Sul (Gordon et al., 2000, Pope; Gallani; Rowntree, 2000). Esse modelo compõe o cenário de emissões A2 do AR4 (IPCC, 2013), sendo esta, então, a ferramenta indicada para os estudos que se apoiam neste relatório.

Diversos centros de pesquisas devolveram modelos de integração de arquivos climáticos para previsão de cenários, sendo o HadCM3 especifico para o uso da ferramenta CCWorldWeatherGen, do Instituto de Pesquisa de Meteorologia (UK).

O CCWorldWeatherGen utiliza o conceito de time-slice para representar os cenários de projeções futuras: time-slice 2020 (período de 2011-2040), time-slice 2050 (período de 2041-2070) e time-slice 2080 (período de 2071-2100).

Diante deste contexto e considerando a tenra idade desta temática científica, este estudo tem por objetivo elaborar um panorama das publicações acerca das projeções de aumento da temperatura da temperatura do ar e do consumo de energia elétrica em edificações, considerando os parâmetros do Quarto Relatório (AR4) do Painel Intergovernamental sobre Mudanças Climáticas (IPCC), por meio de uma revisão sistemática.

\section{MATERIAIS E MÉTODOS}

Adota-se como referencial metodológico, a revisão sistemática de literatura, que consiste em um método para identificar, selecionar, avaliar e sintetizar informações importantes que estão disponíveis acerca de um determinado tema, permitindo realizar uma investigação focada em um tópico definido (GALVÃO e PEREIRA, 2014). Pretende-se, com isso, responder a seguinte pergunta problema: Quais são as projeções das variáveis temperatura interna e consumo de energia nas avaliações de desempenho das edificações sob o efeito do aquecimento global?

As buscas foram com janela temporal de dez anos nas plataformas ScienceDirect e Google Acadêmico. Os descritores foram definidos para direcionarem aos temas das mudanças climáticas, ferramentas de criação de cenários climáticos futuros, arquivos 
climáticos e resiliência das edificações (Tabela 1). Utilizou-se "and" como critério booleano, na relação de todos os descritores.

Tabela 1 - Plataformas de busca e respectivos descritores

\begin{tabular}{c|l|l}
\hline Plataformas de buscas & \multicolumn{1}{c}{ ScienceDirect } & \multicolumn{1}{c}{ Google Acadêmico } \\
\hline & "Climate Change" & "Mudanças climáticas" \\
& "Residential Building" & "Cenários futuros" \\
Descritores & "Climate Change" & "Mudanças climáticas" \\
& "Building Envelope" & "Desempenho termoenergético" \\
& "CCWorldWeather" & \\
& "Residential Building" & \\
& "CCWorldWeatherGen" & \\
& "Weather data file" & \\
\hline
\end{tabular}

As buscas na plataforma ScienceDirect direcionou ao Journal ELSEVIER e aos periódicos Energy Procedia, Building and Environmental, Applied Energy, Energy, Energy and Buildings e Renewable Energy.

A plataforma Google Acadêmico proporcionou acesso aos Repositórios das Universidades e as Bibliotecas Digitais da Universidade de Brasília (UnB), Universidade Federal do Espirito Santo (UFES) e Universidade de São Paulo (USP), ao Laboratório de Eficiência Energética em Edificações (LABEEE), a Revista Ambiente Construído (volume 13) e aos anais dos seguintes eventos científicos: Encontro Nacional de Tecnologia do Ambiente Construído (ENTAC), Encontro Nacional de Conforto no Ambiente Construído (ENCAC) e Encontro Latino-Americano de Conforto no Ambiente Construído (ELACAC).

A seleção sistematizada dos trabalhos foi realizada seguindo os princípios de Dresch, Lacerda e Antunes Junior (2014). Após as buscas nas plataformas, na etapa de pré-avaliação, procedeu-se à leitura dos títulos, resumos e palavras-chaves a fim de verificar a potencialidade das publicações para a finalidade do estudo. As publicações pré-selecionadas foram classificadas como alta, média ou baixa, em três dimensões: qualidade da execução, adequação à questão da revisão e adequação ao foco da revisão (Tabela 2).

Tabela 2 - Critérios de Pós-avaliação

\begin{tabular}{|c|c|c|c|}
\hline \multicolumn{3}{|c|}{ Avaliação do objeto selecionado quanto à } & \multirow{2}{*}{$\begin{array}{c}\text { Critério de ponderação da } \\
\text { qualidade }\end{array}$} \\
\hline Qualidade da execução & $\begin{array}{c}\text { Adequação à questão } \\
\text { da revisão }\end{array}$ & $\begin{array}{c}\text { Adequação ao foco } \\
\text { da revisão }\end{array}$ & \\
\hline Alta & Alta & Alta & Alta \\
\hline Alta & Alta & Média & Média \\
\hline Alta & Média & Média & Média \\
\hline Média & Média & Média & Média \\
\hline Alta & Alta & Baixa & Baixa \\
\hline Alta & Média & Baixa & Baixa \\
\hline Média & Média & Baixa & Baixa \\
\hline Média & Baixa & Baixa & Baixa \\
\hline Baixa & Baixa & Baixa & Baixa \\
\hline
\end{tabular}

Fonte: Adaptado de Dresch, Lacerda e Antunes Junior (2014).

O critério para classificação de uma publicação como alta nos termos do presente estudo considerou o uso da metodologia Morphing para elaboração dos cenários climáticos futuros, tendo como foco a resiliência das edificações por simulações computacionais.

Este procedimento permitiu a análise pós-avaliação, que envolve a consolidação baseada em uma média das dimensões anteriores, selecionando-se as publicações que obtiveram classificação alta nas três dimensões. 
Da Guarda, Emeli; et al; Desempenho Termoenergético de Edificações Sob a Ótica das Mudanças Climáticas: Uma Revisão Sistemática. E\&S - Engineering and Science, 2020, 9:1.

\section{RESULTADOS E DISCUSSÕES}

A etapa de pré-avaliação resultou em 37 artigos, sendo selecionadas 29 publicações na base de dados ScienceDirect e oito no Google Acadêmico, excluindo-se as publicações que constavam em ambas as plataformas (Tabela 3).

Tabela 3 - Descritores e Etapa de pré-avaliação

\begin{tabular}{c|c|c|c}
\hline \multicolumn{5}{c|}{ ScienceDirect / ELSEVIER } & Pré-Classificados \\
\hline Climate change adaptation & $A N D$ & Residential Building & 10 \\
\hline Climate change & $A N D$ & Building envelope & 08 \\
\hline CCWorldWeatherGen & $A N D$ & Residential Building & 06 \\
\hline CCWorldWeatherGen & $A N D$ & Weather data file & 05 \\
\hline \multicolumn{5}{c}{ Google acadêmico } \\
\hline Mudanças climáticas & $A N D$ & Cenários futuros & 02 \\
\hline Mudanças climáticas & AND & Desempenho termoenergético & 06 \\
\hline
\end{tabular}

Na etapa de pós-avaliação foram classificados com qualidade alta sete artigos, a saber: Jorge et al. (2018), Song e Ye (2017); Sabunas e Kanapickas (2017); Wang, Liu e Brown (2017); Invidiata e Ghisi (2016); Triana, Lamberts e Sassi (2016); Casagrande e Alvarez (2013) e uma dissertação: Casagrande (2013) (Tabela 4).

Tabela 4 - Publicações pré-selecionados e critérios de pós-avaliação da qualidade

\begin{tabular}{l|c|c|c|c}
\hline \multicolumn{1}{c|}{ Autores / Ano } & $\begin{array}{c}\text { Qualidade } \\
\text { da } \\
\text { execução }\end{array}$ & $\begin{array}{c}\text { Adequação à } \\
\text { questão da revisão }\end{array}$ & $\begin{array}{c}\text { Adequação ao } \\
\text { foco da revisão }\end{array}$ & $\begin{array}{c}\text { Pós- } \\
\text { avaliação }\end{array}$ \\
\hline Jorge et al. (2018) & Alta & Alta & Alta & Alta \\
\hline Weng (2017) & Alta & Alta & Média & Média \\
\hline Song e Ye (2017) & Alta & Alta & Alta & Alta \\
\hline Ascione, et al. (2017) & Alta & Alta & Média & Média \\
\hline Andric, et al. (2017) & Alta & Alta & Média & Média \\
\hline Sabunas e Kanapickas (2017) & Alta & Alta & Alta & Alta \\
\hline Wang, Liu e Brown (2017) & Alta & Alta & Alta & Alta \\
\hline Costa e Baptista (2017) & Alta & Alta & Média & Média \\
\hline Triana, Lamberts e Sassi (2017) & Alta & Média & Média & Média \\
\hline Tateoka e Duarte (2017) & Alta & Alta & Média & Média \\
\hline Bellido, Fargallo e Arcas (2016) & Alta & Alta & Média & Média \\
\hline Sehizadeh e Ge (2016) & Alta & Alta & Média & Média \\
\hline Hooff, et al. (2016) & Alta & Alta & Média & Média \\
\hline Huang e Hwang (2016) & Alta & Alta & Média & Média \\
\hline Andric et al. (2016) & Alta & Alta & Média & Média \\
\hline Invidiata e Ghisi (2016) & Alta & Alta & Alta & Alta \\
\hline Triana, Lamberts e Sassi (2016) & Alta & Alta & Média & Média \\
\hline Triana, Lamberts e Sassi (2016) & Alta & Alta & Alta & Alta \\
\hline Dirks et al. $(2015)$ & Alta & Média & Média & Média \\
\hline Barbosa, Vicente e Santos & Alta & Alta & Média & Média \\
\hline & & & & Continua
\end{tabular}


Tabela 4 - Publicações pré-selecionados e critérios de pós-avaliação da qualidade

\begin{tabular}{l|c|c|c|c}
\hline \multicolumn{1}{c|}{ Autores / Ano } & $\begin{array}{c}\text { Qualidade } \\
\text { da } \\
\text { execução }\end{array}$ & $\begin{array}{c}\text { Adequação à } \\
\text { questão da revisão }\end{array}$ & $\begin{array}{c}\text { Adequação ao } \\
\text { foco da revisão }\end{array}$ & $\begin{array}{c}\text { Pós- } \\
\text { avaliação }\end{array}$ \\
\hline Hooff et al. $(2015)$ & Alta & Alta & Média & Média \\
\hline Alves e Duarte* (2015) & Alta & Alta & Média & Média \\
\hline $\begin{array}{l}\text { Fahmy, Mahdy e Nikolopoulou } \\
(2014)\end{array}$ & Alta & Alta & Média & Média \\
\hline Hrabovszky-Horváth et al. $(2013)$ & Alta & Média & Baixa & Baixa \\
\hline Jentsch e Bourikas (2013) & Alta & Alta & Média & Média \\
\hline Yu, Chan e Rachel (2013) & Alta & Alta & Média & Média \\
\hline Casagrande e Alvarez (2013) & Alta & Alta & Alta & Alta \\
\hline Casagrande* (2013) & Alta & Alta & Alta & Alta \\
\hline Wan et al. (2012) & Alta & Alta & Média & Média \\
\hline Yildiz et al. $(2012)$ & Alta & Alta & Média & Média \\
\hline Yu, Chan e Rachel (2012) & Alta & Alta & Média & Média \\
\hline Ren, Chen e Wang (2011) & Alta & Alta & Média & Média \\
\hline Wong, Wan, Li e Lam (2010) & Alta & Média & Média & Média \\
\hline
\end{tabular}

*Dissertações

Foram identificadas as localizações geográficas com a finalidade de reconhecer a distribuição espacial dos estudos sobre o tema, identificando os tipos de clima para os quais o método Morphing já foi aplicado. Para cada estudo identificou-se o time-slice com a finalidade de verificar sua coerência com os períodos estabelecidos no AR4, a saber: 2020 (período de 2011-2040), 2050 (período de 2041-2070) e 2080 (período de 2071-2100).

Song e Ye (2017) tomaram como local de estudo a Província de Guangdong, no Sul da China, adotando como time-slice 2020, 2050 e 2080, assim como Jorge et al. (2018) e Indiviata e Ghisi (2016), no Brasil, e por Wang, Liu e Brown (2015), nos Estados Unidos. Todos esses autores não fizeram referência ao período relativo ao time-slice, sendo deduzido que foi o mesmo recomendado no AR4: time-slice 2020 (período de 2011-2040), time-slice 2050 (período de 2041-2070) e time-slice 2080 (período de 2071-2100).

Sabunas e Kanapickas (2017) desenvolveram estudo para Kaunas, na Lituânia. Jorge et al. (2018) para a cidade de Cuiabá (Brasil), Triana, Lamberts e Sassi (2016), para as cidades de São Paulo e Salvador (Brasil). Casagrande (2013), para Vitória (Brasil) e Casagrande e Alvarez (2013), para as cidades de Belém, Recife, Brasília, Vitória, São Paulo e Porto Alegre (Brasil). Todos fazem referência ao time-slice de acordo com o estabelecido no AR4.

Identificaram-se as tipologias das edificações consideradas em cada publicação e as ferramentas utilizadas para a simulação de desempenho termoenergético. Song e Ye (2017), utilizaram os softwares Transient System Simulation Tool (TRNSYS), Sabunas e Kanapickas (2017) utilizaram o software Design Builder e os demais trabalhos utilizaram o software EnergyPlus. As tipologias se referem a residências unifamiliares de padrão médio e de interesse social, multifamiliares e edifícios comerciais.

Jorge et al. (2018) investigaram os impactos das mudanças climáticas no desempenho térmico e no consumo energético de uma habitação de interesse social, localizada no clima tropical de savana. Os autores utilizaram o cenário A2 do IPCC e os time-slice de 2020, 2050 e 2080, aplicaram a metodologia Morphing. O consumo anual de energia da habitação padrão atual de $3.829 \mathrm{kWh} / \mathrm{m}^{2}$, elevou-se para $4.581 \mathrm{kWh} / \mathrm{m}^{2}$ em $2020,4.983 \mathrm{kWh} / \mathrm{m}^{2}$ em 2050 e de $5.498 \mathrm{kWh} / \mathrm{m}^{2}$ em 2080. Os autores concluem que para contrabalancear os efeitos das 
mudanças climáticas estratégias de projeto devem ser incorporadas às novas edificações com vistas a torná-las mais resilientes ao aquecimento global.

Song e Ye (2017) avaliaram duas residências multifamiliares, considerando dois modelos: residência de baixo desempenho (3.5 stars) e residência de alto desempenho (6.5 stars). O consumo total de energia (aquecimento, resfriamento, aquecimento de água, iluminação e equipamentos), conforme padrões de desempenho energético chinês denominado JGJ-75, padrão de design para eficiência energética de edifícios residenciais em zona de verão/ quente e inverno/quente. Utilizaram o cenário A2 do AR4 (IPCC, 2013) e a metodologia Morphing. Os resultados indicaram um aumento da temperatura interna em $0,82^{\circ} \mathrm{C}, 1,91^{\circ} \mathrm{C}$ e $3,41^{\circ} \mathrm{C}$, para os time-slices de 2020,2050 e 2080 , respectivamente, para ambas as residências. Já a demanda de energia apresentou uma elevação de $25 \%$ e $20 \%$, para aquecimento e resfriamento, respectivamente até o time-slice de 2080. Sendo assim, apontam que, para manter o mesmo nível de consumo energético atual é necessário a utilização de equipamentos eficientes, o uso de energias alternativas e renováveis e a melhoria da envoltória das edificações.

Wang, Liu e Brown (2017) investigaram os impactos das mudanças climáticas em um prédio de escritórios de médio porte. Para a integração de arquivos de extensão EPW, utilizaram o modelo climático Hadley Center Coupled Model (HadCM3), para o cenário A2, sendo realizado para cinco cidades americanas: Miami (FL), Phoenix (AZ), Los Angeles (CA), Washington (DC) e Akron (CO), que representam as Zonas Bioclimáticas (ZB) 1A, 2B, 3B, 4A e 5B, respectivamente, conforme a ASHRAE 90.1 (ASHRAE, 2016). Consideraram cinco medidas para mitigação das mudanças climáticas, a fim de diminuir ou neutralizar o aumento de consumo de energia das edificações e para aliviar o aumento da emissão de $\mathrm{CO}_{2}$, a saber: (a) Ajuste da temperatura base interna no sistema de Heating, Ventilating and Air Conditioning (HVAC) dos ambientes de permanência prolongada: modo de operação para resfriamento diurno ajustado de $21,0^{\circ} \mathrm{C}$ para $20,0^{\circ} \mathrm{C}$ e para aquecimento diurno de $24,0^{\circ} \mathrm{C}$ para $25,0^{\circ} \mathrm{C}$; modo de operação para resfriamento noturno ajustado de $15,6^{\circ} \mathrm{C}$ para $12,7^{\circ} \mathrm{C}$ e para aquecimento noturno de $26,7^{\circ} \mathrm{C}$ para $30,0^{\circ} \mathrm{C}$; (b) Horas de operação de HVAC: o horário de funcionamento durante os dias de semana foi ajustado de 06:00h às 22:00h para 06:00 horas às 20:00h; (c) Configuração de fluxo mínimo de ar da Variable air volume (VAV): a configuração base de VAV ajustada de 30\% para 15\%; (d) Combinação das três medidas acima descritas; (e) Uso misto de ventilação: nos horários em que o edifício não opera utilizando o HVAC, a ventilação natural é tomada como prioridade. As simulações das edificações, desconsiderando a adoção das medidas mitigadoras, indicaram aumento do consumo total de energia até o período de 2080 de 12,8\%, em Miami; 14,0\%, em Phoenix; 10,7\%, em Los Angeles, 4,0\%, em Washington DC e 3,7\%, em Akron. Concluem que por meio da implementação de todas as medidas mitigadoras pesquisadas, é possível lidar com os impactos das mudanças climáticas em 2020, sendo, porém, insuficientes como forma de mitigação para os períodos de 2050 e 2080. Apontam que a estratégia de ventilação mista demostra ser uma medida eficaz para reduzir o consumo energético e, consequentemente, mitigar os impactos das mudanças climáticas nas cidades, nos anos de 2020 e 2080, exceto em Los Angeles, em 2080.

Casagrande (2013) investigou o impacto das mudanças climáticas no desempenho termoenergetico das edificações comerciais artificialmente climatizadas, nas cidades brasileiras de Belém, Recife, Brasília, Vitória, São Paulo e Porto Alegre, escolhidas pelo fato de possuírem arquivos climáticos referentes ao período de 1961 a 1990, em formato de EPW. Para a conversão dos dados climáticos atuais em futuros, foi utilizada a ferramenta CCWorldWeatherGen aplicada ao modelo climático global HadCM3, para os time-slices de 2020, 2050 e 2080. A partir do levantamento das características de edifícios comerciais das seis cidades, originou em um edifício hipotético de 11 pavimentos, de forma retangular, com 
12 salas por pavimento e a cobertura em laje protegida por telhado. Adotaram Ocupação de Escritório, com densidade de 0,14 pessoas $/ \mathrm{m}^{2}$; com os usuários exercendo atividade leve de fator metabólico 0.9. O horário de ocupação foi de Segunda a Sexta (ocupação total das 8:00 às $12: 00 \mathrm{~h}$ e das 14:00 às 18:00h; ocupação reduzida a $50 \%$ das $12: 00$ às 14:00h) e aos Sábados, das 08:00 às 12:00h. As cargas de iluminação e de equipamentos foram de 7,0 e $9,7 \mathrm{~W} / \mathrm{m}^{2}$, respectivamente, considerando os ambientes condicionados conforme Regulamento Técnico da Qualidade do Nível de Eficiência de Edifício Comerciais, de Serviços Públicos (RTQ-C). Analisaram o desempenho considerando percentual de aberturas de 50 e $90 \%$, com e sem dispositivos de proteção solar e orientação solar das fachadas maiores a Norte-Sul e Leste-Oeste. Utilizaram a ferramenta EnergyPlus para realizar o desempenho termoenergético do edifício. Quanto à orientação solar, os modelos orientados a Leste-Oeste apresentaram um aumento de $9,5 \%$ em relação aos modelos orientados a Norte-Sul no período atual. Nos períodos futuros, o maior consumo também é na orientação Leste-Oeste, sendo uma diferença de $12,17 \%$, em 2020, de 12,36\%, em 2050 e de 12,07\%, em 2080, em relação à orientação Norte-Sul. Os resultados para o percentual de abertura com $90 \%$ da área da fachada reproduzem um aumento no consumo de energia de 10,21\% em relação aos modelos de 50\% da área da fachada, no período atual. Em 2020, o aumento é de 11,38\%, em 2050, de 11,12\% e em 2080, de 10,84\%. Os modelos sem proteção solar apresentam um aumento de 14,12\% em relação aos modelos com proteção solar no período atual. Nos períodos futuros, o maior consumo permanece nos modelos sem proteção solar, sendo uma diferença de $15,72 \%$ em 2020, de 15,16\% em 2050 e de 14,68\% em 2080, em relação aos modelos com proteção solar. A síntese dos resultados indica uma elevação no consumo de energia total de 10,7\%, 16,9\% e $25,6 \%$, para os time-slices de 2020, 2050 e 2080, respectivamente.

Casagrande e Alvarez (2013) realizaram estudo semelhantes ao de Casagrande (2013), para a cidade de Vitória/ES, ressaltando a importância de se concentrar em estratégias passivas de projeto, como a orientação e massa térmica. Analisaram o edifício hipotético em diferentes situações: orientação de fachada, dimensões das aberturas e presença de dispositivos de proteção solar. Após o processamento das simulações, procederam à análise dos consumos totais anuais com climatização, iluminação, equipamentos e sua evolução ao longo do século XXI. Considerando o conjunto de oito modelos, observaram um aumento médio no consumo anual de 10,7\%, 17,3\% e 26,5\%, para os time-slices de 2020, 2050 e 2080, respectivamente, em relação ao consumo atual. O edifício com a orientação da fachada a Leste e com $90 \%$ de abertura sem proteção solar apresentou o maior consumo de energia nos cenários atual e futuros, quando comparado às fachadas a Norte e a Sul, com $50 \%$ de aberturas com dispositivo de proteção solar.

Por meio da integração de arquivos de climáticos de extensão EPW com o modelo HadCM3, para o cenário A2 do IPCC (IPCC 2013), Triana, Lamberts e Sassi (2016) investigaram os impactos das alterações climáticas no desempenho termoenergético dos projetos desenvolvidos pelo Programa Nacional de Habitação Social (PNHS), em habitação com área útil de $39,75 \mathrm{~m}^{2}$. O estudo foi desenvolvido para as cidades de São Paulo e Salvador. Os arquivos climáticos foram gerados por meio da ferramenta CCWorldWeatherGen, na versão 1.8, para os anos representativos de 2020 e 2050. O método de avaliação de desempenho utilizou como parâmetro o Programa Brasileiro de Etiquetagem de Energia. Sendo assim, foram realizadas para um dia do ano, em duas formas de operação: uso exclusivo de ventilação natural, considerando $20^{\circ} \mathrm{C}$ para operação das janelas, e uso de condicionamento de ar (HVAC) com setup de $25^{\circ} \mathrm{C}$ das 00:00 às 7:00 horas e de $24^{\circ} \mathrm{C}$ das 7:00 às 00:00 hora. Para aquecimento, os autores consideraram-se o setup de $19,5^{\circ} \mathrm{C}$. O coeficiente de desempenho do sistema de refrigeração e aquecimento de 3.24W/W. Foram considerados quatro usuários, sendo dois nos quartos; quatro na sala/estar e um no banheiro. 
Os autores indicam aumento das temperaturas médias externas futuras para a cidade de São Paulo entre $2,0^{\circ} \mathrm{C}$ e $3,0^{\circ} \mathrm{C}$ para os períodos de 2020 e 2050. Para a cidade de Salvador, o aumento previsto é em torno de $2,0^{\circ} \mathrm{C}$, para ambos os períodos. $\mathrm{O}$ indicador Graus-Hora de Resfriamento aumentou de $300 \%$ em 2050 , passando de 2.447 para $7.491^{\circ} \mathrm{Ch}$ na cidade de São Paulo. Em contrapartida, para aquecimento, obteve-se redução de 95\%, para o mesmo período. Com relação ao desconforto por calor, em São Paulo, notou-se elevação de 9,4\% do cenário atual para 21,6\% em 2050, e redução do desconforto por frio de $16,5 \%$ para 1,5\%, em 2050. Em Salvador, o desconforto para calor aumentou de $32 \%$ para $51 \%$, ou seja, $19 \%$ de aumento até 2050. Por fim, os autores ressaltam que para as casas com ventilação natural, forma de operação predominante no Brasil, o problema futuro será a adaptação do usuário ao aumento da temperatura.

Invidiata e Ghisi (2016) analisaram uma Habitação de Interesse Social (HIS) com área útil de 39,44m² , para três cidades brasileiras: Curitiba, Florianópolis e Belém. Para obter o desempenho térmico e a demanda de energia foi utilizando o software EnergyPlus. Para as simulações, considerou-se a taxas de ocupação de quatro e duas pessoas para a sala de estar e quartos, taxa de iluminação foi de 6 e $5 \mathrm{~W} / \mathrm{m}^{2}$ enquanto a taxa metabólica nestes ambientes foi de 60 e $45 \mathrm{~W} / \mathrm{m}^{2}$, respectivamente. A rotina de uso de equipamentos foi estabelecida de 0 à $24 \mathrm{~h}$, enquanto para iluminação para a sala de estar ficou de 16 às $21 \mathrm{~h}$ e dos quartos das 6 às $7 \mathrm{~h}$ e das 22 às 23h. A simulação ocorreu em condição de Naturalmente Ventilada (NV), considerando a temperatura de $20^{\circ} \mathrm{C}$ pra operação das janelas (abrir ou fechar) e na condição de uso de condicionamento artificial (HVAC), sendo o ponto de ajuste de $18,0^{\circ} \mathrm{C}$ para aquecimento e $24,0^{\circ} \mathrm{C}$ para resfriamento, conforme padrões do RTQ-R. Foram analisadas as variáveis de temperatura do ar, umidade relativa do ar e radiação global, nos cenários futuros das três cidades. A temperatura do ar média mensal sofreu um aumento de $4,6^{\circ} \mathrm{C}, 3,6^{\circ} \mathrm{C}$ e $5,1^{\circ} \mathrm{C}$, nas cidades de Curitiba, Florianópolis e Belém, até o time-slice de 2080. Referente à umidade relativa do ar, observou-se redução de 6,2\%, 1,6\% e 14,3\%, em Curitiba, Florianópolis e Belém, até o time-slice de 2080. A radiação horizontal média aumentará de $7,3 \mathrm{Wh} / \mathrm{m}^{2}, 1,1 \mathrm{Wh} / \mathrm{m}^{2}$ e $22,5 \mathrm{Wh} / \mathrm{m}^{2}$, em Curitiba, Florianópolis e Belém, respectivamente, até o time-slice de 2080. A demanda de energia indica que há um aumento ao longo dos anos em comparação com a demanda atual. A demanda de energia de aquecimento e resfriamento é diferente entre as cidades, variando de $20 \mathrm{kWh} / \mathrm{m}^{2}$, em Curitiba, a $102 \mathrm{kWh} / \mathrm{m}^{2}$, em Belém.

Sabunas e Kanapickas (2017) utilizaram o Quinto Relatório (AR5) do IPCC no modelo RCP 2.6 e 8.5 para estimar as emissões de gases de efeito estufa, bem como os parâmetros de temperatura e demanda de energia. Para estimar a projeção futura do aquecimento, utilizaram os cenários RCP 2.6 e RCP 8.5 denominados "Caminhos de Concentração", considerando os time-slices de 2020 (futuro próximo), 2050 (futuro médio) e 2080 (futuro distante). Tais cenários estimam que a temperatura média global do ar aumentará cerca de $1^{\circ} \mathrm{C}$ até o final do século. Vale ressaltar que os cenários são classificados por meio das emissões de Gases de Efeito Estufa (GEE), sendo RCP 2.6 de baixas emissões e o RCP 8.5 de altas emissões. $O$ foco da pesquisa foi investigar a demanda de energia em uma residência comum na Lituânia, estimando o consumo de energia total anual, incluindo a energia necessária para aquecimento e refrigeração para garantir conforto interno de acordo com ASHRAE Standard 55-2013. Utilizaram para a criação dos cenários futuros a ferramenta CCWorldWeatherGen e para simulação de desempenho, o software DesignBuilder. Com relação às mudanças climáticas, houve um aumento de temperatura média de $1,76^{\circ} \mathrm{C}$ para $\mathrm{o}$ cenário RCP 2.6 e de $4,3^{\circ} \mathrm{C}$ para o cenário RCP 8.5, para o time-slice de 2080 . Quanto ao consumo de energia, estimou-se que a demanda anual de resfriamento aumentará de 6,6\% a $7,6 \%$ no cenário RCP 8.5 , e no cenário RCP 2.6, o aumento será pequeno em relação ao consumo atual, de $4,7 \%$ a $5,8 \%$ no consumo anual, para o time-slice de 2080 . Os autores concluem que o aumento da temperatura média global é significante para o aumento do 
consumo de energia dos edifícios, sendo necessário a utilização de estratégias passivas para tornar o edifício energeticamente eficiente.

Considerando o contexto das mudanças climáticas, percebe-se que a temperatura média do ar externo, bem como o consumo de energia são as variáveis analisadas nas publicações sobre os efeitos do aquecimento global para os períodos de 2020, 2050 e 2080. Ressalta-se que em todos os trabalhos, observa-se para as variáveis ambientais exteriores ao edifício, elevação da temperatura média do ar e da radiação global média, bem como redução da umidade do ar. Do ponto de vista das variáveis do edifício, constata-se elevação da temperatura do ar interno dos ambientes, o que reflete na elevação do consumo de energia e no desconforto térmico destes ambientes (Tabela 5).

Tabela 5 - Prognóstico do comportamento das variáveis pesquisadas devido ao impacto das Mudanças Climáticas

\begin{tabular}{|c|c|c|c|c|c|}
\hline \multirow{2}{*}{ Fonte } & \multirow{2}{*}{ Local } & \multirow{2}{*}{ Variável } & \multicolumn{3}{|c|}{ Resultados } \\
\hline & & & 2020 & 2050 & 2080 \\
\hline \multirow{2}{*}{$\begin{array}{l}\text { Jorge et al. } \\
\quad(2018)\end{array}$} & \multirow{2}{*}{ Cuiabá-MT } & $\begin{array}{l}\text { Temperatura Média do ar } \\
\text { externo }\end{array}$ & $1,5^{\circ} \mathrm{C}$ & $3,0^{\circ} \mathrm{C}$ & $+5,9^{\circ} \mathrm{C}$ \\
\hline & & $\begin{array}{c}\text { Consumo de Energia } \\
\text { anual }\end{array}$ & $20 \%$ & $30 \%$ & $44 \%$ \\
\hline \multirow{3}{*}{$\begin{array}{l}\text { Song e Ye } \\
\quad(2017)\end{array}$} & \multirow{3}{*}{$\begin{array}{c}\text { Provincia de Guangdong, } \\
\text { sul da China }\end{array}$} & $\begin{array}{l}\text { Resfriamento atual (4.8\%- } \\
5.5 \%)\end{array}$ & $* * *$ & $* * *$ & $\begin{array}{c}\text { RCP } 8.5(6.6 \%- \\
7.6 \%) \\
\text { RCP2.6 }(4.7 \%- \\
5.8 \%) \\
\end{array}$ \\
\hline & & $\begin{array}{l}\text { Resfriamento atual } \\
(73.3 \%-87.7 \%)\end{array}$ & $* * *$ & $* * *$ & $\begin{array}{c}\text { RCP } 8.5(83 \%- \\
64 \%) \\
\text { RCP2.6 (69.8\%- } \\
86.8 \%) \\
\end{array}$ \\
\hline & & $\begin{array}{l}\text { Temperatura média do ar } \\
\text { externo }\end{array}$ & $+0,82{ }^{\circ} \mathrm{C}$ & $1,91{ }^{\circ} \mathrm{C}$ & $3,41^{\circ} \mathrm{C}$ \\
\hline \multirow{2}{*}{$\begin{array}{l}\text { Sabunas e } \\
\text { Kanaíckas } \\
\text { (2017) }\end{array}$} & \multirow{2}{*}{$\begin{array}{l}\text { Kaunas, Lituânia } \\
\left(54.88^{\circ} \mathrm{N}, 23.88^{\circ} \mathrm{E}\right)\end{array}$} & $\begin{array}{c}\text { Consumo anual de } \\
\text { energia em relação aos } \\
\text { anos } 90\end{array}$ & $\begin{array}{c}\text { RCP } 8.5(13.3 \%- \\
13.5 \%) \\
\text { RCP } 2.6(8.5 \%- \\
10.3 \%)\end{array}$ & $* * *$ & $\begin{array}{c}\text { RCP } 8.5(26.7 \%- \\
29.6 \%) \\
\text { RCP } 2.6(15 \%- \\
15.6 \%)\end{array}$ \\
\hline & & $\begin{array}{l}\text { Temperatura média do ar } \\
\text { externo }\end{array}$ & & & $\begin{array}{c}\text { + } 1,76{ }^{\circ} \mathrm{C} \text { para o } \\
\text { cenário RCP } 2.6 \\
\text { +e de } 4,3^{\circ} \mathrm{C} \text { para o } \\
\text { cenário RCP } 8.5\end{array}$ \\
\hline \multirow{5}{*}{$\begin{array}{l}\text { Wang, Liu e } \\
\text { Brown } \\
(2017)\end{array}$} & Miami & \multirow{5}{*}{$\begin{array}{l}\text { Consumo de Energia } \\
\text { anual }\end{array}$} & $3,80 \%$ & $7,00 \%$ & $12,80 \%$ \\
\hline & Phoenix & & $3,75 \%$ & $7,50 \%$ & $14,00 \%$ \\
\hline & Los Angeles & & $3,65 \%$ & $6,20 \%$ & $10,70 \%$ \\
\hline & Washignton & & $0,90 \%$ & $1,95 \%$ & $4,00 \%$ \\
\hline & Akron & & $0,90 \%$ & $1,00 \%$ & $3,80 \%$ \\
\hline
\end{tabular}


Da Guarda, Emeli; et al; Desempenho Termoenergético de Edificações Sob a Ótica das Mudanças Climáticas: Uma Revisão Sistemática. E\&S

- Engineering and Science, 2020, 9:1.

continuação

Tabela 5 - Prognóstico do comportamento das variáveis pesquisadas devido ao impacto das Mudanças

Climáticas

\begin{tabular}{|c|c|c|c|c|c|}
\hline \multirow{2}{*}{ Fonte } & \multirow{2}{*}{ Local } & \multirow{2}{*}{ Variável } & \multicolumn{3}{|c|}{ Resultados } \\
\hline & & & 2020 & 2050 & 2080 \\
\hline \multirow{15}{*}{$\begin{array}{c}\text { Invidiata e } \\
\text { Ghisi (2017) }\end{array}$} & Belém (PA) & \multirow{3}{*}{ Consumo de Energia anual } & $43 \%$ & $71 \%$ & $112 \%$ \\
\hline & Curitiba (PR) & & $19 \%$ & $56 \%$ & $136 \%$ \\
\hline & Florianópolis (SC) & & $65 \%$ & $112 \%$ & $186 \%$ \\
\hline & Belém & \multirow{3}{*}{$\begin{array}{l}\text { Temperatura média do ar } \\
\text { externo }\end{array}$} & & & $+4,6^{\circ} \mathrm{C}$ \\
\hline & Curitiba & & & & $+3,6^{\circ} \mathrm{C}$ \\
\hline & Florianópolis & & & & $+5,1^{\circ} \mathrm{C}$ \\
\hline & Belém & \multirow{3}{*}{ Umidade Relativa média } & & & $6,2 \%$ \\
\hline & Curitiba & & & & $-1,6^{\circ} \mathrm{C}$ \\
\hline & Florianópolis & & & & $+14,3^{\circ} \mathrm{C}$ \\
\hline & Belém & \multirow{3}{*}{ Radiação Global média } & & & $+7,3 \mathrm{Wh} / \mathrm{m}^{2}$ \\
\hline & Curitiba & & & & $+1,1 \mathrm{Wh} / \mathrm{m}^{2}$ \\
\hline & Florianópolis & & & & $+22,5 \mathrm{Wh} / \mathrm{m}^{2}$ \\
\hline & Belém & \multirow{3}{*}{ Consumo de Energia } & & & $+20 \mathrm{kWh} / \mathrm{m}^{2}$ \\
\hline & Curitiba & & & & \\
\hline & Florianópolis & & & & $+102 \mathrm{kWh} / \mathrm{m}^{2}$ \\
\hline \multirow{9}{*}{$\begin{array}{c}\text { Triana, } \\
\text { Lamberts e } \\
\text { Sassi (2016) }\end{array}$} & \multirow{5}{*}{ São Paulo } & $\begin{array}{l}\text { Consumo para resfriamento } \\
\text { (atual: } 760 \mathrm{Kwh} / \mathrm{m}^{2} *\end{array}$ & $* * *$ & $\begin{array}{c}1.825 \\
\mathrm{Kwh} / \mathrm{m}^{2} *\end{array}$ & $* * *$ \\
\hline & & $\begin{array}{l}\text { Consumo para aquecimento } \\
\text { (atual: } 87 \mathrm{Kwh} / \mathrm{m}^{2} *\end{array}$ & $* * *$ & $4 \mathrm{Kwh} / \mathrm{m}^{2} *$ & $* * *$ \\
\hline & & Temperatura média do ar & $\begin{array}{c}\text { São Paulo: }+2,0^{\circ} \mathrm{C} \mathrm{a} \\
+3,0^{\circ} \mathrm{C}\end{array}$ & & \\
\hline & & $\begin{array}{c}\text { Consumo Total } \\
\text { (equipamentos, iluminação } \\
\text { e AVAC) } 1.763 \mathrm{Kwh} / \mathrm{m}^{2} *\end{array}$ & $2.205 \mathrm{Kwh} / \mathrm{m}^{2} *$ & $\begin{array}{l}2.745 \mathrm{Kwh} / \mathrm{m} \\
2 *\end{array}$ & $* * *$ \\
\hline & & Conforto térmico & & $\begin{array}{c}\text { desconforto } \\
\text { por calor } \\
\text { aumenta de } \\
9,4 \% \text { para } \\
21,6 \% \\
\text { desconforto } \\
\text { por frio reduz } \\
\text { de } 16,5 \% \\
\text { para } 1,5 \%\end{array}$ & \\
\hline & \multirow{4}{*}{ Salvador } & $\begin{array}{c}\text { Consumo para resfriamento } \\
3.675 \mathrm{Kwh} / \mathrm{m}^{2} * \\
\end{array}$ & $* * *$ & $\begin{array}{l}5.043 \mathrm{Kwh} / \mathrm{m} \\
2 *\end{array}$ & $* * *$ \\
\hline & & $\begin{array}{c}\text { Salvador (BA) Consumo } \\
\text { Total (equipamentos, } \\
\text { iluminação e AVAC) } \\
4.591 \mathrm{Kwh} / \mathrm{m}^{2} *\end{array}$ & $* * *$ & $\begin{array}{l}5.595 \mathrm{Kwh} / \mathrm{m} \\
2(\text { ano })\end{array}$ & $* * *$ \\
\hline & & Temperatura média do ar & & $\begin{array}{l}\text { Salvador: } \\
+2,0^{\circ} \mathrm{C}\end{array}$ & \\
\hline & & Conforto térmico & & $\begin{array}{l}\text { desconforto } \\
\text { por calor } \\
\text { aumenta de } \\
32 \% \text { para } \\
51 \%\end{array}$ & \\
\hline \multirow{6}{*}{$\begin{array}{l}\text { Casagrande } \\
\text { (2013) }\end{array}$} & Belém (PA) & \multirow{6}{*}{ Consumo energético anual } & $10,40 \%$ & $15,4 \%$ & $22,80 \%$ \\
\hline & Recife & & $8,30 \%$ & $14,10 \%$ & $22,10 \%$ \\
\hline & Vitoria & & $10,70 \%$ & $17,3 \%$ & $26,50 \%$ \\
\hline & São Paulo & & $11,70 \%$ & $19,3 \%$ & $29,80 \%$ \\
\hline & Porto Alegre & & $10,10 \%$ & $16,4 \%$ & $23,50 \%$ \\
\hline & Brasília & & $14,40 \%$ & $21,4 \%$ & $31,10 \%$ \\
\hline $\begin{array}{l}\text { Casagrande } \\
\text { e Alvarez } \\
(2013)\end{array}$ & Vitoria & Consumo energético anual & $10,70 \%$ & $17,3 \%$ & $26,50 \%$ \\
\hline
\end{tabular}


Ao se considerar um recorte para os edifícios residenciais brasileiros a partir dos prognósticos feitos por Jorge et al. (2018), Invidiata e Ghisi (2017), Triana, Lamberts e Sassi (2016) e Casagrande (2013) para o ano de 2050, e tomando-se como referencia a projeção da demanda brasileira para a classe de consumo residencial de Demanda de Energia 2050 conduzida na nota técnica DEA 13/15 (DEA, 2016), observa-se que as cidades de clima mais quente resultarão em menores consumos de energia (Brasília: 21,4\%, Vitória: 17,3\%, Recife: 14,1\%, Belém: $71 \%$ e Cuiabá: $30 \%$ ), enquanto que nas cidades mais frios (Porto Alegre: 16,4\%, Florianópolis: 112\%, Curitiba: $56 \%$ e São Paulo:19,3\%) em média maior devido a elevação dos graus horas de aquecimento (Figura 1).

Figura 1 - Projeção da elevação da demanda de energia elétrica para edifícios residenciais brasileiros considerando os impactos das Mudanças Climáticas.

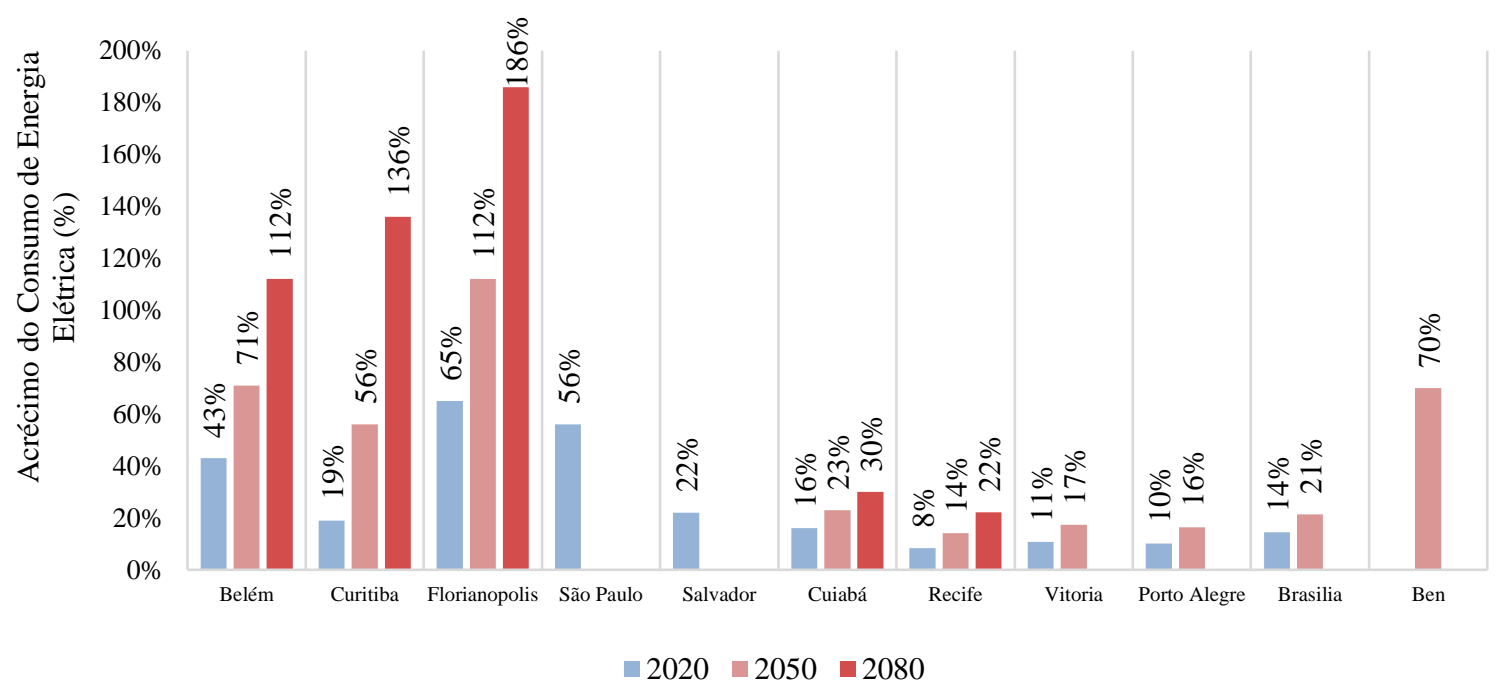

Por outro lado, o aumento da demanda de energia prevista pela Empresa de Pesquisa Energética (EPE) é de $70 \%$, sendo considerado aqui que, embora o número e o consumo unitário dos domicílios tenda a aumentar, ocorrerá um processo de eficientização dos eletrodomésticos (linha branca) e redução do consumo de energia para fins de iluminação, devido ao fim da comercialização de lâmpadas incandescentes e da maior penetração de lâmpadas fluorescentes compactas e de LED (light-emitting diode). O consumo de energia dos principais equipamentos residenciais crescerá, com destaque para o condicionador de ar que, por conta da ampliação do seu acesso pelas famílias, será o principal responsável pelo consumo de energia elétrica nos domicílios.

Nesse sentido, os fatos apresentados indicam que a demanda energética para a classe de consumo residencial, pode ser muito maior que a estimada se considerados os efeitos das mudanças climáticas para a realidade brasileira, em que a maior parte do território apresenta clima tropical predominantemente quente.

\section{CONCLUSÃO}

Ao se considerar os estudos apresentados nesta revisão sistemática, todas as tipologias construtivas demostraram aumento no consumo de energia, em função do aumento das temperaturas externas decorrentes das mudanças climáticas. A prospecção indica que estas temperaturas elevarão entre 1,5 a $5,9^{\circ} \mathrm{C}$ entre 2020 a 2080.

Até o final do século, em Shouthern-China prevê-se um aumento de $25 \%$ e $20 \%$ no consumo de energia para aquecimento e resfriamento, respectivamente. Nas cidades 
americanas, o aumento previsto é de até $10,3 \%$ no consumo total de energia dos edifícios. No Brasil, observa-se elevação no consumo variável ao longo do território nacional em função da zona bioclimática em que a cidade se insere. As cidades inseridas em região de clima tropical com temperaturas elevadas durante quase todo o ano, com exceção de Belém, apresentarão elevação do consumo energético entre 10 a 44\%. Já as cidades localizadas em região de clima Subtropical, como São Paulo, Florianópolis e Porto Alegre, a variação é maior, de $10 \%$ a $186 \%$, provavelmente em virtude da redução dos graus horas de aquecimento, e elevação dos graus horas de resfriamento.

Desta forma, os autores apontam que para a adaptação dos efeitos das mudanças climáticas sob as edificações torna-se necessário a utilização de energias renováveis, uso de equipamentos eficientes e a melhoria das envoltórias das edificações, por meio de estratégias passivas de projeto para proporcionar condições apropriadas de habitabilidade.

\section{REFERÊNCIAS}

ALVES, C. A. Resiliência das edificações às mudanças climáticas na região metropolitana de Sção Paulo. Estudo de caso: Desempenho térmico de edifícios residenciais para idosos. Dissertação (Mestrado), Universidade de São Paulo, São Paulo - SP, 2014.

ANDRIC, I; GOMES, N; PINA, A; FERRÃO, P; FOURNIER, J; LACARRIERE, B; CORRE, L. O. Modeling the long-term effect of climate change on building heat demand: Case study on a district level. Energy and Buildings, v.126, p.77-93, 2016.

ANDRIC, I; PINA, A; FERRÃO, P; FOURNIER, J; LACARRIERE, B; CORRE, O. L. The impact of climate change on building heat demand in different climate types. Energy and Building, v.149, p.225-234, 2017.

ASCIONE, F; BIANCO, N; MASI, R. F. D; MAURO, G. M; VANOLI, G. P. Resilience of robust cost-optimal energy retrofit of buildings to global warming: A multi-stage, multiobjective approach. Energy and Buildings, v.153, p.150-167, 2017.

ASHRAE American Society of Heating, Refrigerating and Air-Conditioning Engineers. Standard 90.1-2016 - Energy Standard for Buildings Except Low-Rise Residential Buildings, 2016.

ASIMAKOPOULOS, D. A; SANTAMOURIS, M; FARROU, I. LASKARI, M; SALIARI, M. ZANIS, G; GIANNAKIDIS, G; TIGAS, K; KAPSOMENAKIS, J; DOUVIS, C; ZEREFOS, S. C; ANTONAKAKI, T; GIANNAKOPOULOS, C. Modelling the energy demand projection of the building sector in Greece in the 21 st century. Energy and Buildings, v.49, p.488-498, 2012.

BARBOSA, R; VICENTE, R; SANTOS R. Climate change and thermal comfort in Southern Europe housing: A case study from Lisbon. Building and Environment, v.92, p.440-451, 2015.

BELCHER, S. E; HACKER, J. N; POWELL, D. S. Constructing desing weather data for future climates. Building Services Engineering Research and Technology, v. 26, p. 49-61. 2005. 
BELLIDO, C. R; FARGALLO, A. P; ARCAS, J. A. P. Otimization of anual energy demand in office buildings under the influence of climate change in Chile. Energy, v.114, p.569-585, 2016.

CASAGRANDE, B. ALVAREZ, C. Preparação de arquivos climáticos futuros para avaliação dos impactos das mudanças climáticas no desempenho termoenergético de edificações. Ambiente Construído, v.13, n.4, p.173-187, 2013.

CASAGRANDE, B. G. Cenários climáticos futuros: Diagnostico prospectivo do desempenho termoenergético de edifícios comerciais no Brasil para o século XXI. Dissertação de Mestrado, Universidade Federal do Espirito Santo-ES, 2013.

COSTA, R. L. Cenários futuros de mudanças climáticas e impactos do conforto térmico humano em áreas urbanizadas. Tese de Doutorado, Universidade de Brasília, Brasília-DF, 2017.

DE VECCHI, R.; SORGATO, M. J.; PACHECO, M.; CÂNDIDO, C.; LAMBERTS, R. Application of the adaptive model proposed by ASHRAE 55 in the Brazilian climate context: Raising some issues. In: 8th Windsor Conference, v. 15251. Anais...Windsor, 2014.

DEMANDA DE ENERGIA (DEA). Nota técnica DEA 13/15. Estudos da Demanda de Energia. Empresa de Pesquisa Energética. Rio de Janeiro, 2016.

DIRKS, J. A; GORRISEN, W. J; HATHAWAY, J. H; SKORSKI, D. C; SCOTT, M. J; PULSIPHER, T. C; HUANG, M; LIU, Y; RICE, J. S. Impacts of climate change on energy consumption and peak demand in buildings: A detailed regional approach. Energy, v.79, p.20-32, 2015.

DRESCH, A.; LACERDA, D. P; ANTUNES JÚNIOR, J. A. V. Design Science Research: Método de Pesquisa para Avanço da Ciência e Tecnologia. Porto Alegre: Bookman, 2014. $204 p$.

DU, H.; EDGE, J.; UNDERWOOD, C. Modelling the impacts of new UK future weather data on a school building. Building Simulation, p. 538-545, 2011.

FAHMY, M; MAHDY, M. M; NIKOLOPOULOU, M. Prediction of future energy consumption reduction using GRC envelope optimization for residential buildings in Egypt. Energy and Buildings, v.70, p.186-193, 2014.

FAHMY, M; MAHDY, M. M; NIKOLOPOULOU. Prediction of future energy consumption reduction using GRC envelope optimization for residential buildings in Egypt. Energy and Buildings, v. 70, p. 186-193, 2014.

Galvão T. F., Pereira M. G. Revisões sistemáticas da literatura: passos para sua elaboração. Epidemiol Serv Saude, v.23, n.1, p.183-184, 2014.

GALVÃO, T. F. Revisões sistemáticas da literatura: passos para sua elaboração. Universidade Federal do Amazonas. Manaus. 2014. 
GORDON, C.; Cooper, C.; Senior, C. A.; Banks, H.; Gregory, J. M.; Johns, T. C.; . Mitchell, J. F. B; Wood, R. A. The simulation of SST, sea ice extents and ocean heat transports in a version of the Hadley Centre coupled model without flux adjustments. Climate Dynamics, v. 16 , p.147-168, 2000.

GOULART, S. V. G. Dados climáticos para avaliação de desempenho térmico de edificações em Florianópolis. 1993. Dissertação (Mestrado). UFSC. Florianopolis: 1993.

HAMDY, M; CARLUCCI, S; HOES, P. J; HENSEN, J. L. M. The impact of climate change on the overheating risk I dwellings - A dutch case study. Building and Environment, v.122, p.307-323, 2017.

HOOF, T. V; BLOCKEN, B. HENSEN, J. L. M; TIMMERMANS, H. J. P. Reprint of: On the predicted effectiveness of climate adaption measures for residential buildings. Building and Environment, v.83, p.142-158, 2015.

HOOF, T. V; BLOCKEN, B; TIMMERMANS, H. J. P; HENSEN, J. L. M. Analysis of the predicted effect of passive climate adaptation measures on energy demand for cooling and heating in a residential building. Energy, v.94, p.811-820, 2016.

HORVATH, S. H; PALVOLGYA, T; CSOKNYAI, T; TALAMON, A. Generalized residential building typology for urban climate change mitigation and adaptation strategies: The case of Hungary. Energy and Buildings, v.62, p.475-485, 2013.

HRABOVSZKY-HORVÁTH S, Pálvölgyi T, Csoknyai T, Talamon A. Generalized residential building typology for urban climate change mitigation and adaptation strategies:

The case of Hungary. Energy Build;v.62, p.475-85, 2013.

HUANG, K. t; HWANG, R. L. Future trends of residential building cooling energy and passive adaptation measures to counteract climate change: The case of Taiwan. Applied Energy, v.184, p.1230-1240, 2016

INVIDIATA, A; GHISI, E. Impact of climate change on heating and cooling energy demand in houses in Brasil. Energy and Building, v.130, p.20-32, 2016.

IPCC - intergovernmental Panel on Climate Change. Understanding Climate Change: 22 years of IPCC assessment, 2013.

JENTSCH, M. F; JAMES, P. A. B; BOURIKAS, L; BAHAJ, A. S. Transforming existing weather data for worldwide locations to anable energy and building performance simulation under future climates. Renewable Energy, v.55, p.514-524, 2013.

JORGE, S. H. M; GUARDA, E. L. A; DURANTE, L. C; CALlEJAS, I. J. A; BLUMENSCHEIN, R. N; ROSSETI, K. A. C. Climate Change Impact on Energy consumption and Thermal performance in low-income houses in Brazilian Savanna. In International Conference for Sustainable Design of the Built Environment - SDBE London, Anais...London, 2018. 
KARIMPOUR, M; BELUSKO, M; XING, K; BOLAND, J; BRUNO, F. Impact of climate change on the design of energy efficient residential building envelopes. Energy and Buildings, v.87, p.142-154, 2015.

KERSHAW, T.; EAMES, M.; COLEY, D. Assessing the risk of climate change for buildings: A comparison between multi-year and probabilistic reference year simulations. Building and Environment, v.46, p.1303-1308, 2011.

LUCIO, P.S.; SILVA, F. D. S.; FORTES, L.T.; SANTOS, L. A. R.; FERREIRA, D.B.; SALVADOR, A. M.; BALBINO, H. T.; SARMANHO, G. F.; SANTOS, L. S. F. C.; LUCAS, E. W. M.; BARBOSA, T. F.; DIAS, P. L. S. Um Modelo Estocástico Combinado de Previsão Sazonal para a Precipitação no Brasil. Revista Brasileira de Meteorologia, v. 25, p. 70-87, 2010 .

POPE, V. D.; GALLANI, M. L.; ROWNTREE, P. R.; STRATTON, R. A. The impact of new physical parametrizations in the Hadley Centre climate model: HadAM3. Climate Dynamics, v. 16, p. 123-146, 2000.

REN, Z; CHEN, Z; WANG, X. Climate change adaptation pathways for Australian residential bilding. Building and Environment, v.46, p.2398-2412, 2011

REPELLI, C. A; ALVES, J. M. B. A variabilidade pluviométrica no setor norte do Nordeste e os eventos El Niño-Oscilação Sul (ENOS). Revista Brasileira de Meteorologia, v.7, n.2, p. 583-592, 1996.

ROBERT, A.; KUMMERT, M. Designing net-zero energy buildings for the future climate, not for the past. Building and Environment, v.55, p.150-158, 2012.

SABUNAS, A. KANAPICKAS, A. Estimation of climate change impacto $\mathrm{n}$ energy consumption in a residential building in Kaunas, Lithuania, using HEED software. Energy Procedia, v.128, p.92-99, 2017.

SEHIZADEH, A; GE, H. Impacto f future climates on the durability of typical residential wall assemblies retrofitted to the passive haus for the Eastern Canada region. Building and Environment, v.97, p.111-125, 2016.

SONG, X; YE, C. Climate change adaptation pathways for residential buidings in Southern China. Energy Procedia, v.105, p.3062-3067, 2017.

SRES - Emissions Scenarios: A Special Report of IPCC Working Group III. Published for the Intergovernmental Panel on Climate Change, ISBN: 92-9169-113-5, 2010.

TATEOKA, S. S; DUARTE, D. H. Adaptação às mudanças climáticas: Desafios para requalificação de edifícios existentes em São Paulo. In Encontro Nacional de Conforto no Ambiente Construído e Encontro Latino-Americano de Conforto no Ambiente Construído. Anais... Balneário Camboriú - SC, 2017. 
TRIANA, M. A; LAMBERTS, R. SASSI, P. Desempenho de Habitações de interesse social frente às mudanças climáticas. In: Encontro Nacional de Tecnologia do Ambiente Construido, 16, 2016, São PAULO. Anais... Porto Alegre: ANTAC, 2016.

VAN HOOFF T, Blocken B, Hensen JLM, Timmermans HJP. Reprint of: On the predicted effectiveness of climate adaptation measures for residential buildings. Build Environ, v.83, p.142-58.

VAN HOOFF T, Blocken B, Timmermans HJP, Hensen JLM. Analysis of the predicted effect of passive climate adaptation measures on energy demand for cooling and heating in a residential building. Energy, v.94, p.811-20, 2016.

WAN, K. K. W; LIN, D. H. W; PAN, W; LAM, J. C. Impact of climate change on building energy use in different climate zones and mitigation and adaptation implications. Applied Energy, v.97, p.274-282, 2012.

WANG, L; LIUI, X; BROWN H. Prediction of the impacts of climate change on energy consumption for a medium-size office building with two climate models. Energy and Buildings, v. 157, p.218-226, 2017.

WENG, K. Perfomance of UK dwelling in projected future climates. Energy Procedia, v.105, p.3727-3732, 2017.

WILDE, P. de; COLEY, D. The implications of a changing climate for buildings. Building and Environment, v.55, p.1-7, 2012.

WONG, S. L; WAN, K. K. W; LI, D. H. W; LAM, J. C. Impact of climate change on residential building envelope cooling loads in subtropical climates. Energy and Buildings, v.42, p.2098-2103, 2010.

XAVIER, T. M. B. S. et al. Previsão regional de chuva para o Estado do Ceará através do projeto "Tempo de Chuva": 1964-1999. Revista Brasileira de Recursos Hídricos, v.5, p.6992. 2000.

YILDIZ, Y; KORKMAZ, K. OZBALTA, T. G; ARSAN, Z. D. An approach for developing sensitive design parameter guidelines to reduce the energy requirements of low-rise apartment buildings. Applied Energy, v.93, p.337-347, 2012.

YU, F. W; CHAN, K. T; SIT, R. K. Y. Climate influence on the design and operation of chiller systems serving office buildings in a subtropical climate. Energy and Buildings, v.55, p.500-507, 2012.

YU, F. W; CHAN, K. T; SIT, R. K. Y; YANG, J. Energy simulation of sustainable air-cooled chiller system for commercial buildings under climate change. Energy and Buildings, v.64, p.162-171, 2013. 